\section{$\underset{\substack{\text { hommes } \\ \text { \& migrations }}}{ }$}

\section{Hommes \& migrations}

Revue française de référence sur les dynamiques

migratoires

$1288 \mid 2010$

Langues et migrations

\title{
Langue maternelle et langue d'écriture
}

\section{Martine Paulin}

\section{(2) OpenEdition \\ Journals}

\section{Édition électronique}

URL : http://journals.openedition.org/hommesmigrations/878

DOI : 10.4000/hommesmigrations.878

ISSN : 2262-3353

\section{Éditeur}

Musée national de l'histoire de l'immigration

\section{Édition imprimée}

Date de publication : 1 novembre 2010

Pagination : 118-128

ISSN : 1142-852X

Référence électronique

Martine Paulin, «Langue maternelle et langue d'écriture », Hommes \& migrations [En ligne], 1288 | 2010, mis en ligne le 29 mai 2013, consulté le 19 avril 2019. URL : http://journals.openedition.org/

hommesmigrations/878; DOI : 10.4000/hommesmigrations.878 


\section{Langue maternelle et langue d'écriture}

Par Martine Paulin, agrégée de Lettres modernes, Cité nationale de l'histoire de l'immigration, département Éducation

Le français comme langue d'écriture questionne des identités en chantier, façonnées par l'Histoire. Ce choix linguistique est un détour nécessaire voire obligé, pour dire dans une langue des mondes qui

l'excèdent. À cheval entre deux univers de sens, les écrivains francophones développent l'art de se jouer des frontières. Certains questionnent les liens avec leur terre natale quand d'autres retournent, dans la langue, les armes conçues pour la dominer. Derrière l'abandon de la langue maternelle se profile sa présence irréductible qui continue de hanter le langage de l'autre. 
“Je parle une autre langue : qui suis-je ?' Dans L'Arbre à dires ${ }^{(1)}$, avec ce titre de chapitre, Mohammed Dib ouvre une interrogation fondamentale : changer de langue, est-ce changer d'identité, ne plus être soi, devenir autre ? De quelle identité s'agit-il, de quelle élaboration multiple : historique ? sociale ? culturelle ? familiale ? individuelle ? Comment le travail d'écriture, lorsqu'on est un auteur venu d'ailleurs, marque-t-il cette quête?

Nombreux sont les écrivains immigrés qui tentent de définir leur relation à cette problématique : le dialogue entre langue maternelle et langue d'écriture s'avère le plus souvent chaotique, oscillant entre séduction et bannissement, réconciliations et tensions, avec l'espoir, voire le fantasme, de conquérir un équilibre apaisé. Une source de tourments et d'exaltation créatrice... Pour quelles raisons abandonne-t-on sa langue maternelle? Pour quelles raisons y retourne-t-on? Que dire des écrivains issus de pays colonisés par la France et dont la langue, ou l'une des langues, est déjà le français ?

\section{Quitter le territoire de la langue maternelle}

Pour certains, quitter définitivement le pays natal, c'est devoir abandonner avec son territoire celui de la langue, dans une volonté de rupture avec le passé, en particulier pour des raisons politiques. Le changement d'identité linguistique s'impose alors, tout comme l'installation en France répond le plus souvent à un choix revendiqué de démocratie et de culture. C'est le cas d'Eduardo Manet, né en 1930 à Santiago de Cuba. En 1968, l'approbation par Fidel Castro de l'invasion des troupes soviétiques en Tchécoslovaquie indigne l'écrivain qui rompt avec Cuba et s'installe définitivement en France. Laisser la langue maternelle, c'est alors pour lui, "glisser dans la peau du français" et devenir un être nouveau. Vassilis Alexakis inscrit son exil dans la même optique : refusant la dictature des Colonels, il quitte la Grèce en 1968, s'installe en France et opte pour la langue du pays d'accueil : "Les livres que je rêvais d'écrire ne pourraient pas parâ̂tre dans mon pays. Je me suis mis à les rêver en français. Je lisais systématiquement les auteurs qui avaient choisi de s'exprimer dans une langue étrangère : Beckett, Nabokov, Conrad. Je les trouvais excellents ${ }^{(2)}$." La motivation pour ces auteurs, notons-le, n'est pas seulement politique, elle est aussi éditoriale. Sans possibilité de retour, il s'agit de conquérir un public, d'investir de nouveaux circuits d'édition, d'échapper aux difficultés de la traduction et donc de devenir français, non seulement comme citoyen mais littérairement.

Pour d'autres, le français comme langue d'écriture de l'exil politique est bien plus qu'un nouvel ancrage : c'est une force de libération idéologique. Atiq Rahimi, écrivain et cinéaste, né en 1962 à Kaboul (en Afghanistan) et réfugié politique en France en 1984, 
s'en explique à propos de Syngué sabour, Pierre de patience, son premier roman écrit en français : "Ma langue maternelle, le persan, m’impose des tabous, des interdits. La langue maternelle dit l'intime, c'est elle qui nous apprend la vie, l'amour, la souffrance, elle qui nous ouvre au monde. C'est aussi la langue de l'autocensure. [...] Avec le français, jétais libéré de tonnes de contraintes affectives. Jusqu'en 2002, quand je suis retourné dans mon pays après dix-huit ans d'exil, jétais incapable d'écrire en français. Je retrouve donc mon pays, ma culture, ma langue, et là, mystère, je ne pouvais plus écrire en persan (3)." Le français, pour l'écrivain afghan, instaure une distanciation libératrice avec les interdits. C'est grâce à elle que pourra se mener le combat contre la barbarie, au prix, il est vrai d'un certain clivage. Pour Mohamed Kacimi, né en Algérie, venu en France en 1981, "le partage est clair" : "Écrire en français, c'est oublier le regard de Dieu et de la tribu, inventer ma marge illusoire mais vitale, mon espace intime, forger ma solitude et ma mémoire, réaliser la rupture avec cette longue chaîne de traditions, d'héritages, de legs, que les miens assument depuis des millénaires. C'est nier le dogme pour célébrer toute transgression. Je n'écris pas en français. J'écris en 'moi-même ${ }^{(4) . "}$

\section{Le détour par l'Autre et la question de l'identité}

Les raisons politiques ou idéologiques ne sont pas les seules : adopter une nouvelle langue, c'est aussi partir en conquête, vouloir renaître. François Cheng, né en 1929 en Chine et venu en France en 1949, revendique ce corps à corps dans Le Dialogue $e^{(5)}$ : "En m’investissant totalement dans le français, je me voyais obligé de m'arracher à ce quifaisait mon passé et d'effectuer le plus grand écart que constitue le passage d'une écriture idéographique [...] à une écriture phonétique [...]. Cet arrachement et cet écart, ne m'ayant pas fait me perdre en chemin, m'auront permis de me ré-enraciner, non seulement dans ma terre d'accueil, ce qui est déjà beaucoup pour un exilé, mais proprement dans l'être, puisque par cette nouvelle langue, jai accompli l'acte, je le répète, de nommer à neuf les choses, y compris mon propre vécu." Le choix de la langue répond ici au défi de l'intégration, à la conquête d'une place nouvelle, il est vécu comme une richesse culturelle, sans reniement du passé.

Pour certains écrivains, l'adoption d'une langue d'écriture nouvelle protège d'une double appartenance qui peut fragiliser : devant la nostalgie qui menace, mieux vaut couper le cordon pour ne pas être "deux en soi". La langue d'origine, parce qu'elle exhibe l'intimité, peut exposer l'individu, le mettre en péril : la langue d'adoption est alors préférée, à la fois comme barrière et libération. Mais il arrive aussi que le passage d'une langue à l'autre ne génère ni force ni refus, soit vécu comme une perte et aboutisse à un sentiment de deuil, ainsi que l'explique Agota Kristof. Venue de Hongrie, arrivée en Suisse en 1956, adoptant la langue française comme langue 
d'écriture, elle écrit dans L'Analphabète: "Je parle le français depuis plus de trente ans, je l'écris depuis vingt ans, mais je ne le connais toujours pas [...]. C'est pour cette raison que jappelle la langue française une langue ennemie, elle aussi. Il y a encore une autre raison, et c'est la plus grave : cette langue est en train de tuer ma langue maternelle $e^{(6)}$."

Devant cette menace de dépression identitaire, le retour actif à la langue maternelle, sous des formes multiples, peut faire figure de retour aux sources et de reconstruction.

\section{Reconquérir la langue maternelle}

Revenir à la langue maternelle, c'est lui redonner un territoire, un public, une légitimité politique: Kateb Yacine, écrivain né en 1929 en Algérie, immigré en France dans les années cinquante, revient dans son pays après l'Indépendance. Après avoir écrit son ceuvre en français, langue coloniale officielle, il renoue de manière militante, dans les années soixante-dix, avec la langue parlée de sa région d'origine, en écrivant en arabe dialectal des pièces de théâtre populaire.

Retrouver la langue maternelle, c'est aussi ouvrir le champ au travail de mémoire, pour ressusciter en version originelle les émotions et sensations du passé, sans la médiation de la traduction, sans sa quête obsessionnelle de coïncidence. Eduardo Manet l'indique à propos de Mauricio, personnage de La Sagesse du singe : : Quand l'espagnol s'immisce dans ses rêves, il entrâ̂ne avec lui des sensations en châ̂ne, l'impression d'ouvrir les tiroirs les plus secrets de sa mémoire ; des odeurs qui n'appartiennent qu'au pays de sa naissance, des visages disparus ; le goût d'un fruit qu'il aime, dont il ne se rappelle ni la forme ni la couleur ; des voix familières et douces ; une chanson, disons plutôt une berceuse." C'est pourquoi la madeleine proustienne de la langue, clé d'une mémoire sensorielle et émotive, qui ramène pour beaucoup à l'enfance et à la jeunesse, s'inscrit souvent dans un temps clivé. Pour Nedim Gürsel, né en 1951 en Turquie et venu en France à la suite du coup d'État de 1971, les langues ne se partitionnent pas aussi nettement sur la frontière du temps. Le refuge de la langue maternelle, comme il l'explique dans Les Mots de l'exil, subit au présent les assauts du français :

"Bien que jhabite Paris depuis près de vingt ans, jai l'impression d'habiter la cave où la lampe de Kafka reste toujours allumée. À vrai dire je n'habite pas une ville ni un pays, mais une langue. Le turc est ma cave où je suis dans l'écriture comme le noyau dans le fruit. J'écris donc dans ma langue maternelle et cela me rassure. Pourtant je suis traversé dans ma vie quotidienne par la langue française qui me hante. Parfois, elle parvient à briser les murs de ma cave et déclenche dans mon écriture un mécanisme irréversible, une sorte de déchirure ${ }^{(8)}$." La langue maternelle figure ici comme lieu protecteur, mais de manière ambivalente : si la cave et le fruit protègent en effet des agressions extérieures et nourrissent 
l'inspiration, ils sont aussi des métaphores de l'enfermement. Le français qui fait irruption devient alors un agent à la fois menaçant et libérateur.

D'où la nécessité pour certains de faire une sorte d'“autoanalyse" linguistique" afin de se débarrasser des "larves" du passé, image empruntée à Dumitru Tsepeneag, écrivain roumain qui confie dans Le Mot sablie $e^{(9)}$ : "Aussi dois-je continuer pour le moment à écrire en roumain pour me débarrasser enfin de ce ballast fantasmatique : car qui me garantit que je ne me retrouverai pas hanté par tous ces spectres....] Il serait louche en creusant pour moi neuf de la langue française que j'en exhume des cadavres d'images venues d'une autre terre, avec un autre horizon ${ }^{(10)}$."

Ces positions, nous le voyons, reflètent le caractère conflictuel du processus. C'est pourquoi il arrive que le choix comme l'abandon d'une langue deviennent impossibles : faire coexister langue maternelle et langue d'écriture, c'est refuser d'être scindé, refuser la coupure entre passé et présent, étranger et français, ancien public et nouveau public, ancienne identité, nouvelle identité... C'est vouloir rester "uni en soi", si l'on peut dire. Pratiquer le bilinguisme, dont le territoire se décline de manière variée, nous allons le voir, c'est ne pas avoir à faire un deuil angoissant.

\section{La question du bilinguisme}

Andreï Makine arrive d'URSS en France en 1987 et obtient l'asile politique. L'auteur, en linguiste et idéologue, pratique simultanément dans un même texte le français et le russe, avec changement de langue d'un personnage à l'autre, travail sur le lexique ou les connotations. "Linterrogation sur l'identité est, souvent, la source ou du moins le corollaire d'un changement de langue, qui n'a pas pour seule vocation d'exprimer un refus, mais bien de répondre à un désir de reconstruction. Se définir par rapport à l'Autre, avec les moyens de l'Autre, n'est-ce pas encore tenter de se définir, sous un jour renouvelé, par rapport à Soi et à ses Origines ${ }^{(11)}$ ?"

D'autres déclinaisons du bilinguisme existent, non pas à l'intérieur d'une ceuvre, mais d'ceuvre à ceuvre, notamment dans la pratique de l'autotraduction. L'exemple de Milan Kundera est de ce point de vue intéressant : né en Tchécoslovaquie en 1929, cet écrivain, croyant à la réforme du communisme et acteur du Printemps de Prague, s'est vu obligé, avec l'intervention des troupes du pacte de Varsovie en 1968, de quitter la Tchécoslovaquie. Il s'est alors exilé en France, a écrit tout d'abord en langue tchèque, puis directement en français dans la seconde moitié des années quatre-vingt. Lisant les traductions de ses originaux écrits en tchèque, il se rend compte qu'on a corrigé, "réécrit" son ceuvre. Milan Kundera va donc soumettre à la révision toutes ses productions et s'autotraduire. De ce fait, il se sensibilise à la question des équivalences 
lexicales, des nuances et connotations culturelles des mots, interrogeant, dans les deux sens, le fondement même du travail artistique. Il n'est pas le seul à souligner ainsi la créativité que les écrivains immigrés doivent à leur vigilance linguistique, au fait que pour eux, la langue ne soit pas "naturelle".

Avec Samuel Beckett, le phénomène de l'autotraduction est plus complexe encore. Né à Dublin en 1906, après une enfance passée dans sa famille, des séjours à Paris et des retours en Irlande, il s'établit à Paris en 1937. C'est à partir de 1944, mais surtout dans les années cinquante et ceci jusqu'à sa mort, que Beckett écrit une ceuvre bilingue, hanté, dans les deux langues, par la dimension du néant, obsédé par la nomination. Qu'est-ce que parler? Nommer? Comment rendre la hantise fascinée de la disparition, de la dilution dans le soliloque, dans le silence? Le véritable personnage de Beckett, c'est le Langage. Il faut se souvenir de L'Innommable:

\section{Toutes ces options esthétiques et culturelles, qu'elles soient de coexistence des langues dans un même texte, d'autotraduction ou d'unilinguisme, peuvent être difficiles à vivre pour certains.} le personnage ne peut pas bouger, il ne peut pas parler, ni se taire. Il va disparaître dans les mots : il n'identifie pas les mots, ce sont les mots qui l'identifient... "Il faut dire des mots, tant qu'il y en a, il faut les dire, jusqu'à ce qu'ils me trouvent, jusqu'à ce qu'ils me disent, étrange peine, étrange faute, il faut continuer, c'est peut-être déjà fait, ils m'ont peut-être déjà dit, ils m'ont peut-être porté jusqu'au seuil de mon histoire, devant la porte qui s'ouvre sur mon histoire, ça m'étonnerait, si elle s'ouvre, ça va être moi, ça va être le silence, là où je suis, je ne sais pas, je ne le saurai jamais, dans le silence on ne sait pas, il faut continuer, je ne peux pas continuer, je vais continuer ${ }^{(12)}$." Dans son livre La Bataille du soliloque, Chiara Montini écrit à propos de la langue de Beckett que c'est "une langue qu'il a toujours voulu étrangère, écrivant d'abord en français à la place de l'anglais, langue maternelle, et retournant à l'anglais quand le français lui devenait trop familier afin d'éviter les automatismes d'une langue maternelle, et donner au verbe tout son pouvoir de nomination, c'est-à-dire de création ${ }^{(13) "}$. C'est donc l'auteur par excellence qui n'abandonne ni sa langue maternelle, ni sa langue d'accueil, qui quitte l'une quand elle ne lui paraît plus suffisamment étrange - plutôt qu'étrangère - et inversement, et qui les explore indéfiniment. Une démarche quasi métaphysique, bien au-delà du thème de l'exil puisqu'elle nous transporte au fond de ce que parler veut dire.

Toutes ces options esthétiques et culturelles, qu'elles soient de coexistence des langues dans un même texte, d'autotraduction ou d'unilinguisme, peuvent être difficiles à vivre pour certains. L'image du cordon ombilical, qu'on voudrait couper ou préserver, revient constamment dans les écrits : si la langue est dite "maternelle", c'est qu'elle renvoie au continent charnel premier, réveillant au passage bien des affects. 


\section{La mère dans la langue}

C'est la mère qui rappelle le caractère ineffaçable de la langue comme empreinte et marquage généalogique, symbolique, culturel, affectif. Sarah Lévi-Lopez, dans La Sagesse du singe ${ }^{(14)}$ d'Eduardo Manet, explique à son fils ce qu'est le ladino, langue de ses ancêtres : "La langue des juifs sefardim, mon fils. Le judéo-espagnol qu'ils ont emporté avec eux dans leur exode. Cette langue, je la garde dans mon ceur comme un secret précieux. Quatre, cinq, dix siècles plus tard, on n'oublie pas, Mauricio, on n'oublie jamais. [...] L'exil est un voyage qui n'en finit pas. Lêtre humain n'est pas un caméléon qui peut facilement changer de peau." Ici, la langue, c'est le corps, la chair, l'être. Eduardo Manet, comme bien d'autres, avec son enthousiasme pour la langue française, reste sensible aux différents échos générationnels de l'appartenance linguistique, dont il sait qu'elle est toujours beaucoup plus que cela. Aujourd'hui, il envisage d'écrire en espagnol un livre d'hommage à sa mère... Autre exemple : après le décès de sa mère en 1993, Vassilis Alexakis, renoue avec le grec, en même temps qu'il rend hommage dans La Langue maternelle ${ }^{(15)}$ à celle qui l'avait mis en garde contre le délaissement de sa langue. En quête de réconciliation entre les deux langues, alors qu'au départ, comme nous l'avons vu plus haut, il avait opté de manière "innocente" pour le français, l'écrivain traduit ses romans du français vers le grec, et plutôt que de traduction, il faudrait parler de réécriture. C'est par ce retour, presque à rebours vers le ventre maternel, que l'auteur tente aussi de dompter le non-dit dont il ne peut nier l'existence : "J'ai le sentiment qu'il existe des silences entre les phrases, de longs silences, comme si jécrivais pour taire une chose plutôt que pour l'exprimer." Quant à Samuel Beckett, exilé par nécessité vitale, il lui fallait couper le cordon avec une mère à l'amour rigide et autoritaire qui lui inflige de fréquentes querelles, c'est de la mort de celle-ci, en 1950, que date la véritable émancipation du bilinguisme, c'està-dire, non pas une ceuvre de traduction, mais d'écriture en deux versions.

Sur ce versant de la haine-amour cedipienne, on peut évoquer, mais cette fois-ci sur le plan véritablement clinique, un certain Louis Wolfson, né en 1931 : auteur du livre Le Schizo et les Langues, il est américain, mais son livre est écrit en français. Le narrateur schizophrène explique, à la troisième personne, comment il fuit compulsivement sa langue-mère: "Comme ce n'était guère possible que de ne point écouter sa langue natale, il essayait de développer des moyens d'en convertir les mots presque instantanément [...] en des mots étrangers chaque fois après que ceux-là pénétreraient à sa conscience en dépit de ses efforts de ne pas les percevoir. Cela pour qu'il pût s'imaginer en quelque sorte qu'on ne lui parlât pas cette maudite langue, sa langue maternelle, l'anglais ${ }^{(16) . "}$

Si certains comme Atiq Rahimi coupent le cordon ombilical pour éviter, cette fois-ci par amour, une relation quasi incestueuse avec la langue ("Ne serait-ce que le mot 'maternel' : il crée trop de liens. Adopter une autre langue, le français, c'est choisir la liberté. 
On ne se marie pas avec sa mère ${ }^{(17),}$, écrit-il), on peut remarquer que bon nombre d'auteurs se placent dans un équilibre instable, tantôt apaisés, tantôt en conflit, et que le choix peut être traversé par la culpabilité. Pour les écrivains nés dans les pays colonisés par la France, la culpabilité est encore plus complexe.

\section{La langue mère dans les pays colonisés}

Dans les pays qui ont été colonisés par la France, le français était langue officielle. C'était celle de l'école et des institutions. L'arabe ou le berbère dans les pays du Maghreb, par exemple, étaient infériorisés parce qu'appartenant aux "indigènes" et notamment perçus comme étrangers au processus artistique et culturel. En Afrique, certaines langues, dont la pratique n'est qu'orale, ne peuvent se substituer au français écrit. C'est ce qu'explique l'écrivain togolais Sami Tchak, né en 1960 et arrivé en France en 1986, à propos de sa langue maternelle, le tem ou kotokoli. Pour lui, la question est de parvenir à "traduire" un univers africain en français, en gardant dans le style l'oralité de la langue d'origine. On retrouve le même désir chez Léonora Miano, née au Cameroun en 1973 et installée en France depuis 1991 : “Ma langue d'auteur n'est un français classique qu'en apparence. [...] Ma ponctuation n'est pas toujours orthodoxe. Elle cherche des rythmes non européens. Il y a toujours, dans le soubassement de la phrase, une multitude d'autres langues. Celles dans lesquelles je ne pense pas, mais que je ressens. J'écris dans l'écho des cultures qui m'habitent : africaine, européenne, afro-américaine, caribéenne. Tout cela vient naturellement se loger dans le texte. Mon esthétique est donc frontalière ${ }^{(18)}$."

Alain Mabanckou, né en 1966 au Congo Brazzaville, insiste également sur ce point : "Cela fait partie de ma propre culture puisque la plupart des langues africaines que je parle sont orales. N'ayant pas de base écrite, elles n'existent qu'à travers cette oralité. Et là-dessus vient se greffer la langue française comme langue d'écriture. Finalement, dans Mémoires de porc-épic, la langue est française, mais le rythme est congolais. C'est celui de mon ethnie, de ma tribu. De ce rythme dans la langue provient toute l'oralité du récit. Il y a un peu d'ailleurs dans ce français-la ${ }^{(19)}$." Aux périodes troublées de la colonisation, la question de la langue, depuis plus apaisée, a été vive. Le français, c'est alors la langue du colon. C'est, comme le dit Charles Bonn, universitaire spécialiste des littératures du Maghreb, "l'instrument d'une profonde blessure identitaire autant que politique. Le choix de cette langue est parfois vécu comme celui de la capitulation, et il est à l'origine celui des pères défaillants dans leur rôle de garants de la loi que représente la langue ${ }^{(20)}$."

L'universitaire cite à ce propos Le Polygone étoilé de Kateb Yacine, où le père du futur écrivain, pourtant fin lettré en arabe, veut le convaincre qu'il faut entrer à l'école 
française. Et le fils raconte comment il épouse, devant sa mère, le désir de capitulation de son père: "Ma mère était trop fine pour ne pas s'émouvoir de l'infidélité qui lui fut ainsi faite. Et je la vois encore, toute froissée, m'arrachant à mes livres - tu vas tomber malade! puis un soir, d'une voix candide, non sans tristesse, me disant: 'Puisque je ne dois plus te distraire de ton autre monde, apprends-moi donc la langue française...' Ainsi se referma le piège des Temps Modernes sur mes frêles racines, et j’enrage à présent de ma stupide fierté, le jour où, un journal français à la main, ma mère s'installa devant ma table de travail, lointaine comme jamais, pâle et silencieuse [...]. Jamais je n'ai cessé, même aux jours de succès près de l'institutrice, de ressentir au fond de moi cette seconde rupture du lien ombilical, cet exil intérieur qui ne rapprochait plus l'écolier de sa mère que pour les arracher, chaque fois un peu plus, au murmure du sang, aux frémissements réprobateurs d'une langue bannie, secrètement, d'un même accord, aussitôt brisé que conclu... Ainsi avais-je perdu tout à la fois ma mère et son langage, les seuls trésors inaliénables - et pourtant aliénés ! ${ }^{(21) "}$.

\section{Le pouvoir de l'écriture face au poids de l'Histoire}

Devant cette capitulation qui peut être perçue comme une sorte de collaboration culturelle et politique, Kateb Yacine une fois devenu écrivain brandira le français comme un "butin de guerre" : "La francophonie est une machine politique néocoloniale, qui ne fait que perpétuer notre aliénation, mais l'usage de la langue française ne signifie pas qu'on soit l'agent d'une puissance étrangère, et j’écris en français pour dire aux Français que je ne suis pas français", déclare-t-il en 1966. Et ce butin de guerre, cet engagement, seront donc, pour subvertir la culpabilité, des réponses à la mère patrie désirée - l'Algérie algérienne -, aussi bien qu'à la mère génitrice.

Pour Assia Djebar, née en Algérie en 1936, vivant entre ce pays et la France depuis 1962, un peu comme pour Kateb Yacine, le français est une arme, celle qui permet notamment de porter la parole des femmes du Maghreb : écrire en français, c'est "surgir hors d'une pénombre". Pouvoir lutter, protester, donner vie et rendre hommage, en devenant à son tour celle qui transmet. Un combat politique et féministe. Dans Ces Voix qui m'assiègent, l'auteure écrit : "Écrire se fait aujourd'hui, pour moi, dans une langue, au départ, non choisie, dans un écrit français qui a éloigné de ce fait l'écrit arabe de la langue maternelle ; cela aboutit, pour moi, non pas à ma voix déposée sur papier, plutôt à une lutte intérieure avec son silence porteur de contradictions et qui s'inscrit peu àpeu ou d'emblée dans l'épaisseur d'une langue, la plus légère, la plus vive ou n'importe laquelle! Simplement mise à disposition; dans mon cas, le français. [...] Peut-être même, pendant longtemps, me suis-je sentie portée le plus souvent par des voix non françaises - elles qui me hantent et qui se 
trouvaient être souvent voix ennemies du français, puisque celui-cifut si longtemps langue de l'occupant. [...] Oui, ramener les voix non francophones - les gutturales, les ensauvagées, les insoumises - jusqu'à un texte français qui devient enfin mien. [...] Oui, faire réaffleurer les cultures traditionnelles, mises au ban, maltraitées, longtemps méprisées, les inscrire, elles, dans un texte nouveau, dans une graphie qui devient 'mon' français ${ }^{(22)}$."

La situation de Leila Sebbar est particulière : née en 1941 d'un père algérien et d'une mère française, tous deux instituteurs en Algérie, elle ne parle pas l'arabe, la langue de son père et du peuple colonisé, et écrit dans la langue de sa mère. "J'écris des romans avec de l'étranger", dit-elle. Son ceuvre est hantée par ce questionnement qui devient une quête d'identité aux accents cedipiens. Il s'agit d'atteindre la langue du père (interdite, tue) et de la traduire, de la faire vivre dans la langue de la mère, comme deux corps de naissance brouillés par l'histoire coloniale et qu'il faut reconquérir. Elle écrit dans L'Arabe comme un chant secret : "J'offre à mon père non pas son peuple sur sa terre et dans sa langue mais des fragments du corps algérien dans le silence de l'exil, dans l'exil de l'autre langue et de son école hospitalière, sur la rive française de sa femme revenue au pays natal sans avoir jamais quitté sa langue. [...] Mon père a donné ses enfants à sa femme, à la France, à la langue d'amour qui l'a reçu comme maître d'école modèle, il lui a donné le meilleur et sa jeunesse, ses élans d'idéalisme républicain, malade de justice et d'égalité. Il ne pouvait pas être ce Juste dans la langue de sa mère? Ou elle était là, présente en scur jumelle, et je ne le savais pas? Je ne le sais pas? Je traduis l'Algérie, je traduis mon père dans la langue de ma mère. Je lui fabrique, je me fabrique une famille immense des deux côtés de la mer. Je crois ainsi rétablir une filiation rompue ${ }^{(23) . "}$

Une multitude de pratiques d'écriture, dont bien d'autres exemples pourraient être cités, illustrent la bivalence linguistique: entrelacement, interpénétration, autotraduction, travail stylistique exigeant. Souvent, une langue "fait irruption" dans l'autre, si l'on peut dire... Dans cette dialectique complexe, c'est non seulement la création artistique, mais aussi la quête de soi qui est en jeu. Au cceur de l'écriture se joue un positionnement identitaire, politique, culturel, dans sa dimension cedipienne et mémorielle, avec ses fractures, conscientes ou non, au sein de l'individu, des fratries et de la société. La question de la langue et du style est au cceur de toute ceuvre littéraire : le français comme langue d'écriture intensifie chez les écrivains en exil leur conscience artistique et existentielle.

\section{Bibliographie}

- Amati Melher Jacqueline, Argentieri Simona, Canestri, Jorge, La Babel de linconscient: langue maternelle, langues étrangères et psychanalyse, Paris, PUF, 1994 (épuisé, consulter BNF).

- Ben Jelloun Tahar, Suis-je un écrivain arabe ?, 28/11/2004, site officiel de l'auteur, rubrique "Chroniques". http://www.taharbenjelloun.org 
- Bonn Charles, Littérature francophone. Tome 1 : Le Roman, ouvrage collectif sous la direction de Charles Bonn et Xavier Garnier, Paris, Hatier, 1997.

- Casnav de Créteil, conférences d'écrivains : "Langue maternelle, langue d'écriture : parcours d'écrivains". http://casnav.ac-creteil.fr

- Delbart Anne-Rosine, Les Exilés du langage. Un siècle d'écrivains français venus d'ailleurs (1919-2000), Limoges, Pulim, coll. Francophonies, 2005.

- Djebbar Assia, L'Amour, la Fantasia, Paris, Le Livre de Poche, 2001 (première édition 1985).

- Fleischer Alain, L'Accent, une langue fantôme, Paris, Les Petits Matins, 2006.

- Gasquet Axel et Suárez Modesta, L'Hospitalité des langues : écrivains multilingues et écritures métisses, Clermont-Ferrand, Presses Universitaires Blaise Pascal, 2004.

- Gatti Stéphance, Kateb Yacine, un poète en trois langues, Montreuil, La Parole Errante, Paris, 1994.

- Hamad Nazir, La Langue et la Frontière, double culture et polyglottisme, Paris, Denoël, 2004.

- Huston Nancy, Nord perdu, Mazamet, Babel, 1999.

- Molina Romero Carmen, "Double langue et création littéraire", in Revue Imaginaire \$ Inconscient, n 14, 2004.

- Montini Chiara, La Bataille du soliloque, Genèse de la poétique bilingue de Samuel Beckett (1929-1946), Amsterdam \& New York, Rodopi, coll. Faux Titre, 2007.

- Oustinoff Michaël, Bilinguisme d'écriture et auto traduction - Julien Green, Samuel Beckett, Vladimir Nabokov, Paris, L'Harmattan, coll. Critiques Littéraires, 2001.

- Perisanu Mariana, "Écrivains français d'origine roumaine et leur rapport à la langue", in Actes de la XXI biennale de la langue française, 2005.

- Sebbar Leïla, Je ne parle pas la langue de mon père, Paris, Julliard, 2003.

\section{Notes}

1. Dib Mohammed : L'Arbre à dires, Paris, Albin Michel, 1998.

2. Alexakis Vassilis, Les Mots étrangers, Paris, Stock, 2002.

3. Propos recueillis par Martine Laval pour Télérama, mars 2009.

4. Kacimi Mohamed, L'Orient après l'amour, Paris, Actes Sud, 2008.

5. Cheng François, Le Dialogue, Paris, Desclée de Brouwer, coll. Proches Lointains, 2002.

6. Kristov Agota, L'Analphabète, Genève, Zoé, 2004.

7. Manet Eduardo, La Sagesse du singe, Paris, Grasset, 2001.

8. Gürsel Nedim, "Les Mots de l'exil", in Le Dernier Tramway, trad. A.M Toscan du Plantier, Paris, Seuil, 1991.

9.T sepeneag Dumitru, Le Mot sablier, Paris P.O.L., 1984.

10. Graphie et ponctuation respectées.

11. Makine Andreï, Le Testament français, Paris, Gallimard, 1995.

12. Beckett Samuel, L'Innommable, Paris, Les Éditions de Minuit, 1953.

13. Montini Chiara, La Bataille du soliloque, Genèse de la poétique bilingue de Samuel Beckett (1929-1946),

Amsterdam \& New York, Rodopi, coll. Faux Titre, 2007.

14. Manet Eduardo, op. cit.

15. Alexakis Vassilis, La langue maternelle, Paris, Fayard, 1995.

16. Wolfson Louis, Le Schizo et les langues, Paris, Gallimard, 1970.

17. Propos recueillis par Martine Laval pour Télérama, mars 2009

18. Miano Léonora : site officiel de l'auteure, rubrique "sensibilité". www.leonoramiano.com

19. Propos recueillis par Mathieu Menossi pour Evene.fr, 2007.

20. Bonn Charles, "Le roman maghrébin", in Littérature francophone. Tome 1 : Le Roman, ouvrage collectif sous la direction de Charles Bonn et Xavier Garnier, Paris, Hatier, 1997.

21. Kateb Yacine, Le Polygone étoilé, Paris, Seuil, 1966.

22. Djebar Assia, Ces voix qui m’assiègent, Paris, Albin Michel, 1999.

23. Sebbar Leïla, L'Arabe comme un chant secret, Saint-Pourçain-sur-Sioule, Bleu autour, 2007. 